\title{
FUNCTIONAL STRENGTH EXERCISE AND BLOOD-FLOW RESTRICTION PROTOCOLS: A SYSTEMATIC REVIEW
}

\author{
Nicole J. Hernandez, , A, B, D Bradley J. Myers, , A, D Yuri Feito, , , A, D Jennifer A. Bunn 1, A, B, D \\ ${ }^{1}$ Campbell University, Physical Therapy, Buies Creek, NC, United States \\ ${ }^{2}$ Kennesaw State University, Exercise Science and Sport Management, Kennesaw, GA, United States \\ A Study Design; ${ }^{\mathrm{B}}$ Data Collection; ${ }^{\mathrm{D}}$ Manuscript Preparation \\ Address for correspondence: \\ Jennifer Bunn \\ PO Box 1090, Buies Creek, \\ NC 27506, 910-893-1361 \\ E-mail: bunnj@campbell.edu
}

\begin{abstract}
Ahstract Objective: To assess current literature focused on the influence of functional training with blood flow restriction (BFR) on muscular adaptation in adults below the age of 65 years.

Methods: A systematic literature search was performed with the following databases: PubMed, CINAHL Complete EBSCOhost, and ScienceDirect. Two researchers filtered the articles according to the criteria and quality, supported by the Consolidated Standards of Reporting Trials (CONSORT) tool. Studies were limited to those with participants with a mean age between 18 and 65 years. Articles were peer-reviewed, available in English, and utilized either multi-joint resistance exercises or functional exercise as the intervention.

Results: The search yielded 16 studies on uninjured, older adult, injured and athletic subjects. Regardless of the population, the studies tended to favor BFR training for improvements of strength, but this was highly dependent on cuff pressure and training load.

Conclusions: Current literature suggests that BFR with functional and or multi-joint strength training is successful in improving strength, hypertrophy, function and in pain reduction. Favorable results with functional BFR included procedures using relative and well-controlled occlusion pressure. More research is necessary to understand the effect of BFR on self-reported outcomes and hypertrophy in athletic and older adults.
\end{abstract}

Key worlds resistance training, arterial occlusion, low intensity, muscle hypertrophy

\section{Introduction}

Blood-flow restriction (BFR) training, also known as arterial occlusion training, utilizes low-intensity movements as a way to stimulate musculoskeletal adaptation and provides an alternative method to traditional heavy-load resistance training (Loenneke, Wilson, Marin, Zourdos, Bemben, 2012; Takarada, Takazawa, Sato, Takebayashi, Tanaki, Ishii, 2000). Many studies have confirmed the positive effect of BFR on increasing muscular hypertrophy and strength, similar to traditional resistance training (Cook, Kilduff, Beaven, 2014; Giles, Webster, McClelland, 
Cook, 2017; Godawa, Credeur, Welsch, 2012; Loenneke, Wilson, Marin, Zourdos, Bemben, 2012; Madarame, Ochi, Tomioka, Nakazato, Ishii, 2011; Ozaki et al., 2013; Takarada, Tsuruta, Ishii, 2004; Teramoto, Golding, 2006; Yasuda, Fujita, Ogasawara, Sato, Abe, 2010). With traditional resistance training for the general population, the American College of Sports Medicine (ACSM) guidelines suggest a frequency of 2-3 days per week at an intensity of 60$70 \%, 1$ repetition maximum (1RM) and 8-12 repetitions, to achieve muscle adaptation over a 6-8 week period (Garber et al., 2011). BFR training requires a frequency of 2-3 days per week at a reduced intensity of $30-50 \%$, 1RM and 15-30 repetitions, resulting in muscle adaptations in as little as 3-5 weeks (Cook et al., 2014; Credeur, Hollis, Welsch, 2010; Garber et al., 2011; Loenneke et al., 2012; Takarada et al., 2000; Takarada et al., 2004). Lowintensity high repetition training without BFR does not elicit substantive improvements in muscular strength and hypertrophy, as it does not provide the hypoxic environment necessary to promote the appropriate metabolic stress for these gains (Hughes et al., 2018; Loenneke et al., 2012). Further, traditional resistance training is often difficult in a rehabilitation setting due to patient limitations in completing a high number of repetitions or going to failure.

Research has shown the benefits of BFR training in many populations, such as: older adults (Araujo et al., 2015; Segal, N., Davis, Mikesky, 2015; Segal, N.A., Williams, Davis, Wallace, Mikesky, 2015), individuals who are recovering from injury or surgery (Giles et al., 2017; Hughes et al., 2018; Tennent et al., 2017), and those who cannot tolerate high-intensity resistance training (Araujo et al., 2015; Giles et al., 2017; Hughes et al., 2018; Segal, N. et al., 2015; Segal, N.A. et al., 2015; Tennent et al., 2017). This is relevant in a healthcare setting, where injured and older adult patients are seeking muscular adaptation during rehabilitation, but are unable to follow ACSM guidelines. Although BFR strength gains may be delayed and the magnitude of these gains are lower than in highintensity resistance training, BFR training may provide a safe alternative to achieve therapeutic goals (Brandner, May, Clarkson, Warmington, 2018; Hughes et al., 2018; Loenneke et al., 2012; Patterson et al., 2019).

Much of the early BFR research included single-joint isotonic exercises in males, with results strongly focused on strength and hypertrophy (Hughes et al., 2018; Lixandrao et al., 2018). More contemporary BFR work has expanded to a variety of populations, such as females (Araujo et al., 2015; Credeur et al., 2010; Giles et al., 2017; Segal, N. et al., 2015; Teramoto, Golding, 2006), those with kidney disease (Clarkson et al., 2017), chronic fatigue syndrome (McCully, Smith, Rajaei, Leigh, Natelson, 2003), and older adults (Araujo et al., 2015; Segal, N. et al., 2015; Segal, N.A. et al., 2015). Most studies in these populations have focused on improvements in hypertrophy and strength, with limited transference into function in these populations, unless exercises are salient and specific (Pelletier, Higgins, Bourbonnais, 2015). The use of BFR as a clinical rehabilitation tool has been established (Hughes et al., 2018), but further carry-over into function, multi-joint exercises and strength improvements is lacking. Specifically, research related to BFR training and functional outcomes such as gait speed, sit-to-stand, balance, and timed up and go, are often conducted with participants over 65 years old (Clarkson et al., 2017). Expanding to younger populations, few studies have captured how BFR could be implemented in a manner which elicits reductions in pain at rest and with movement, subjective and functional outcome measures, and subjective quality of life. Therefore, the purpose of this systematic review was to assess current available literature focused on the influence of functional and multi-joint BFR training on muscular adaptation and improvement in adults below the age of 65 years. 


\section{Materials and Methods \\ Search strategy}

A literature search was performed to gather high-quality peer-reviewed research papers concerning functional BFR training. The following keywords were utilized to search the PubMed, CINAHL Complete EBSCOhost and ScienceDirect databases: ("vascular occlusion" OR "kaatsu" OR "blood flow restrict") AND ("strength training" OR "low-load" OR "low-intensity" OR "resistance training" OR "exercise training" OR "function" OR "weight lifting" OR "walking OR cycling"), and ("vascular occlusion" OR "kaatsu" OR "blood flow restrict" OR "BFR") AND ("strength training" OR "low-load" OR "low intensity" OR "resistance training" OR "function" OR "athlete" OR "pain" OR "performance" OR "improve"). Search filters for all databases covered: January 2004-January 2019, all adult, academic journals, English, peer-reviewed, human, and randomized control trials. The most recent 15 years of literature was chosen because this includes the bulk of BFR literature. The search was limited to adults below the age of 65 years to remove the potential effects of aging on the musculature.

\section{Inclusion and exclusion critteria}

Research studies were included for review if the population was human, participant mean age was between 18 years and 65 years, were peer-reviewed, available in English, and they utilized functional or multi-joint resistance exercises (e.g. functional exercise) as the intervention. Studies were excluded if they only used single-joint resistance exercises not related to a task or functional movement, or only utilized BFR during recovery from another exercise or intervention, participants were older than 65 years, or they included a population outside of the inclusion criteria.

\section{Study selection}

The total search from all three databases yielded 840 articles. Irrelevant and duplicate articles were removed, shortening the list to 34 articles $(n=34)$. Researchers NH and JB removed nine further studies $(n=25)$ that focused on aerobic conditioning, as well as ten $(n=15)$ that utilized practical BFR methods with elastic straps and little control for measuring occlusion. The final article removed $(n=14)$ focused on the effects of BFR on detraining, which was deemed irrelevant for this review. Figure 1 depicts the search process. The Consolidated Standards of Reporting Trials (CONSORT) tool was utilized to help discriminate which high-quality studies were to be included. The tool was helpful in identifying the study's design, criteria, randomization, blinding, attrition rate with reasoning, limitations, and generalizability. Randomized controlled trials (RCTs) were preferred, but studies were not excluded if they used a different study design. 


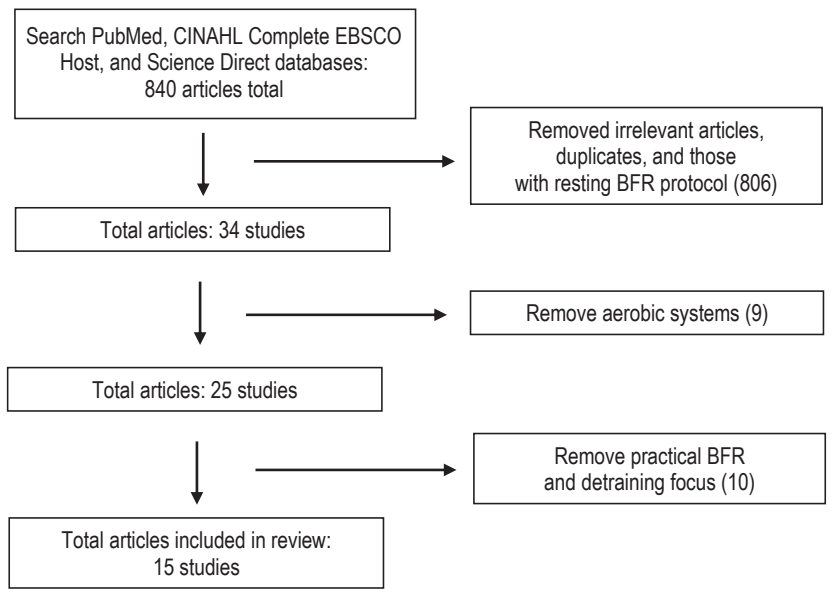

Figure 1. Flow chart of selection research article process for systematic review

\section{Resullts}

The sixteen ( $n=16$ ) studies reviewed were grouped into four different population categories: uninjured young adult $(n=7)$, older adult $(n=3)$, rehabilitation $(n=3)$, and athletes $(n=2)(n=15)$. Grouping was utilized to better differentiate the outcomes of functional BFR training across multiple populations, and to clarify gaps in literature. All studies are organized by population of interest in Table 1.

Table 1. Summary of articles organized by population of study. Age is represented as mean (SD), and studies were conducted in active individuals unless otherwise noted. RCT is randomized control trial, BFR is blood flow restriction, 1RM is one-rep max, MVC is maximal voluntary contraction, CSA is cross-sectional area, CMJ is counter movement jump, $\mathrm{HI}$ is high intensity, Ll is low intensity, $C B$ is combination of high and low intensity training, TUG is timed up and go, ACL is anterior cruciate ligament, $A D L$ is activities of daily living, PT is physical therapy, KOOS is Knee Injury and Osteoarthritis Outcome Score, VR12 is Veterans RAND 12-item Health Survey, $\mathrm{PCr}$ is phosphocreatine

\begin{tabular}{|c|c|c|c|c|}
\hline Author, year & $\begin{array}{l}\text { Participants: } \mathrm{n} \text {-size, } \\
\% \text { male, age, any } \\
\text { other special IC/EC }\end{array}$ & $\begin{array}{l}\text { Study design } \\
\text { and groups }\end{array}$ & $\begin{array}{l}\text { Methods: length of intervention, BFR cuff procedures, } \\
\text { reps, sets, load }\end{array}$ & Outcomes \\
\hline 1 & 2 & 3 & 4 & 5 \\
\hline \multicolumn{5}{|c|}{ Uninjured young adult population } \\
\hline $\begin{array}{l}\text { D.P. Credeur } \\
\text { et al., } 2010\end{array}$ & $\begin{array}{l}\mathrm{N}=12,41.7 \% \\
\text { male, } 22(1) \mathrm{yrs}\end{array}$ & $\begin{array}{l}\text { RCT; subjects served } \\
\text { as own control; } \\
\text { trained BFR side vs } \\
\text { control side }\end{array}$ & $\begin{array}{l}\text { 4-wk training, } 3 \text { sessions/wk; } 60 \% \text { MVC handgrip } \\
15 \times / \text { minute for } 20 \mathrm{~min} \text { at } 80 \mathrm{~mm} \mathrm{Hg} \text { or no occlusion }\end{array}$ & $\begin{array}{l}\text { MVC and forearm circumference: } \\
\text { BFR and control increased, } \\
\text { but no group differences. } \\
\text { Blood artery flow-mediated dilation: } \\
\text { Control > BFR }\end{array}$ \\
\hline $\begin{array}{l}\text { H. Madarame } \\
\text { et al., } 2011\end{array}$ & $\begin{array}{l}\mathrm{N}=17,100 \% \text { male, } \\
19.9(1.6) \text { yrs, } \\
\text { Untrained young } \\
\text { men }\end{array}$ & $\begin{array}{l}\text { Groups allocated } \\
\text { for similarity; } \\
\text { no randomizing } \\
\text { or blinding; BFR } \\
\text { vs normal training } \\
\text { groups }\end{array}$ & $\begin{array}{l}\text { 10-wk training, } 2 \text { sessions/wk; Both groups performed } \\
30-40 \% 1 \mathrm{RM} \text { leg press } 30-15-15-15 \mathrm{reps} \text {; BFR started } \\
\text { at } 200 \mathrm{mmHg} \text { and increased to } 250 \mathrm{mmHg} \text { as program } \\
\text { progressed }\end{array}$ & $\begin{array}{l}\text { Squat: BFR > control . } \\
\text { CSA: both groups increased, } \\
\text { but no group differences. } \\
\text { CMJ: no significant change } \\
\text { with training }\end{array}$ \\
\hline
\end{tabular}




\begin{tabular}{|c|c|c|c|c|}
\hline 1 & 2 & 3 & 4 & 5 \\
\hline $\begin{array}{l}\text { H. Ozaki et al., } \\
2013\end{array}$ & $\begin{array}{l}\mathrm{N}=19,100 \% \text { male, } \\
23.5 \text { (1) yrs }\end{array}$ & $\begin{array}{l}\text {, RCT; Groups: BFR, } \\
\text { HI group }\end{array}$ & $\begin{array}{l}\text { 6-wk training, } 3 \text { sessions/wk of bench press; } \mathrm{HI}: 3 \times 10 \\
\text { reps at } 75 \% 1 \mathrm{RM} \text {; BFR: } 30-15-15-15 \text { reps at } 30 \% 1 \mathrm{RM} \text {; } \\
\text { BFR started at } 100 \mathrm{mmHg} \text { for first session and increased } \\
\text { by } 10 \mathrm{mmHg} \text { each session until } 160 \mathrm{mmHg} \text { pressure } \\
\text { was reached }\end{array}$ & $\begin{array}{l}\text { Bench press, arm CSA, chest } \\
\text { CSA: both groups increased, } \\
\text { but no group differences. } \\
\text { Decreased carotid arterial } \\
\text { compliance: } \mathrm{HI}>\text { BFR }\end{array}$ \\
\hline $\begin{array}{l}\text { M. Teramoto, } \\
\text { L.A. Golding, } \\
2006\end{array}$ & $\begin{array}{l}N=19,45 \% \text { male, } \\
23.9(4.5) \text { yrs, } \\
\text { College students }\end{array}$ & $\begin{array}{l}\text { RCT; cross-over } \\
\text { design; trained } \\
\text { BFR side vs control } \\
\text { side; examiners } \\
\text { counterbalanced } \\
\text { dominant and non- } \\
\text { dominant legs among } \\
\text { participants }\end{array}$ & $\begin{array}{l}\text { 5-wk training, } 3 \text { sessions/wk; Step exercise of } 2 \times 5 \text {-min } \\
\text { step exercise was performed each session; BFR was set } \\
\text { at mean occlusion pressure of } 95.4(6.9) \mathrm{mmHg}\end{array}$ & $\begin{array}{l}\text { Leg press strength: BFR > control. } \\
\text { Muscular endurance, muscle size: } \\
\text { both groups increased, but no } \\
\text { group differences }\end{array}$ \\
\hline $\begin{array}{l}\text { T. Yasuda } \\
\text { et al., } 2010\end{array}$ & $\begin{array}{l}\mathrm{N}=10,100 \% \text { men. } \\
25.7(5.1) \mathrm{yrs}\end{array}$ & $\begin{array}{l}\text { RCT; groups: BFR, } \\
\text { control }\end{array}$ & $\begin{array}{l}\text { 2-wk training, } 2 \text { sessions/d, } 6 \mathrm{~d} / \mathrm{wk} \text {; Groups performed } \\
30-15-15-15 \text { reps at } 30 \% 1 \mathrm{RM} ; \mathrm{BFR} \text { started at } 100 \\
\mathrm{mmHg} \text { and increased by } 10 \mathrm{mmHg} \text { increments each } \\
\text { session until } 160 \mathrm{mmHg} \text { was reached }\end{array}$ & $\begin{array}{l}\text { Bench press: BFR > control. } \\
\text { Muscle thickness: BFR > control } \\
\text { Anabolic hormones: no change } \\
\text { in either group }\end{array}$ \\
\hline $\begin{array}{l}\text { T. Yasuda } \\
\text { et al., 2011b }\end{array}$ & $\begin{array}{l}\mathrm{N}=40,100 \% \text { men, } \\
23.8(2.5) \mathrm{yrs}\end{array}$ & $\begin{array}{l}\text { RCT; groups: HI, } \\
\text { LI-BFR, CB, non- } \\
\text { training control }\end{array}$ & $\begin{array}{l}\text { 6-wk training, } 3 \text { sessions/wk of bench press; } \mathrm{HI}: 3 \times 10 \\
\text { reps at } 75 \% 1 \mathrm{RM} \text {; LI-BFR: } 30-15-15-15 \text { reps at } 30 \% \\
\text { 1RM; CB: performed 2×/week LI-BFR and } 1 \times / \text { week HI; } \\
\text { BFR started at } 100 \mathrm{mmHg} \text { and increased by } 10 \mathrm{mmHg} \text { at } \\
\text { each session until } 160 \mathrm{mmHg} \text { was reached }\end{array}$ & $\begin{array}{l}\text { Bench press: } \mathrm{HI} \text { and } \mathrm{CB}>\mathrm{LI}-\mathrm{BR} \text {. } \\
\text { Muscle size: groups increased, } \\
\text { but no group differences }\end{array}$ \\
\hline
\end{tabular}

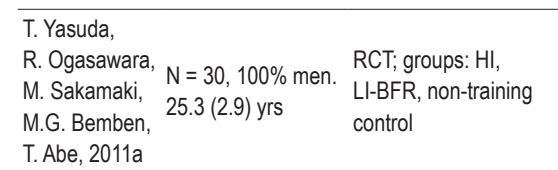

6-wk training, 3 sessions/wk of bench press; $\mathrm{HI}: 3 \times 10$ reps at $75 \%$ 1RM; LI-BFR: $30-15-15-15$ reps at $30 \%$ 1RM; BFR started at $100 \mathrm{mmHg}$ and increased by 10 $\mathrm{mmHg}$ at each session until $160 \mathrm{mmHg}$ was reached
Bench press: $\mathrm{HI}$ and LI-BFR > control.

Muscle CSA: HI and LI-BFR > control

\begin{tabular}{|c|c|c|c|c|}
\hline \multicolumn{5}{|c|}{ Older adult population } \\
\hline $\begin{array}{l}\text { J.P. Araujo } \\
\text { et al., } 2015\end{array}$ & $\begin{array}{l}\mathrm{N}=28,0 \% \\
\text { male. } 54(4) \text { yrs, } \\
\text { postmenopausal } \\
\text { women }\end{array}$ & $\begin{array}{l}\text { RCT; groups: water } \\
\text { exercise, water + } \\
\text { BFR, non-exercise } \\
\text { control }\end{array}$ & $\begin{array}{l}\text { 8-wk training, } 3 \text { sessions/wk; Groups performed } 30-15- \\
15-15 \text { reps with water resistance exercises. BFR at } 80 \% \\
\text { arterial occlusion }\end{array}$ & $\begin{array}{l}\text { Knee extension: water + BFR > } \\
\text { water. } \\
\text { TUG: both groups improved, but } \\
\text { no significant group difference }\end{array}$ \\
\hline $\begin{array}{l}\text { N. Segal et al., } \\
2015\end{array}$ & $\begin{array}{l}\mathrm{N}=44,100 \% \text { men, } \\
56.1(7.7) \text { yrs, } \\
\text { community-dwelling } \\
\text { adult men }\end{array}$ & $\begin{array}{l}\text { RCT; groups: BFR, LI } \\
\text { control }\end{array}$ & $\begin{array}{l}\text { 4-wk training, } 3 \text { sessions/week with bilateral leg press; } \\
\text { BFR: } 30-15-15-15 \text { reps at } 30 \% 1 \mathrm{RM} \text {; Control: } 30-15- \\
\text { 15-15 reps at } 30 \% 1 \mathrm{RM} \text {; BFR started at } 30 \mathrm{mmHg} \text { and } \\
\text { increased throughout session and program until final } \\
\text { exercise pressure of } 200 \mathrm{mmHg} \text { was reached }\end{array}$ & $\begin{array}{l}\text { Leg press: both groups improved, } \\
\text { but no significant group difference. } \\
\text { Pain improvement: } L I \text { > BFR }\end{array}$ \\
\hline $\begin{array}{l}\text { N.A. Segal } \\
\text { et al., } 2015\end{array}$ & $\begin{array}{l}\mathrm{N}=45,0 \% \text { men, } \\
55.3(5.5) \text { yrs, } \\
\text { community-dwelling } \\
\text { adult women }\end{array}$ & $\begin{array}{l}\text { RCT; groups: BFR, LI } \\
\text { control. }\end{array}$ & $\begin{array}{l}\text { 4-wk training, } 3 \text { sessions/week with bilateral leg press; } \\
\text { BFR: } 30-15-15-15 \text { reps at } 30 \% 1 \mathrm{RM} \text {; Control: } 30-15- \\
15-15 \text { reps at } 30 \% 1 \mathrm{RM} \text {; BFR started at } 30 \mathrm{mmHg} \text { and } \\
\text { increased throughout session and program until final } \\
\text { exercise pressure of } 200 \mathrm{mmHg} \text { was reached }\end{array}$ & $\begin{array}{l}\text { Leg press: BFR > LI. } \\
\text { Knee extensor strength: BFR > LI. } \\
\text { Muscle CSA, power, pain: both } \\
\text { groups improved, but no significant } \\
\text { group difference }\end{array}$ \\
\hline \multicolumn{5}{|c|}{ Rehabilitation Setting } \\
\hline $\begin{array}{l}\text { L. Giles et al., } \\
2017\end{array}$ & $\begin{array}{l}\mathrm{N}=69,38 \% \text { male, } \\
27.7(5.5) \text { yrs, } \\
\text { patellofemoral pain } \\
\text { syndrome }\end{array}$ & $\begin{array}{l}\mathrm{RCT} \text {; groups: low } \\
\text { intensity BFR, HI } \\
\text { standard control }\end{array}$ & $\begin{array}{l}\text { 8-wk training; } 3 \text { sessions/week of leg press and leg } \\
\text { extension; HI } 3 \times 7-10 \text { reps at } 70 \% 1 \text { RM; BFR: } 30-15-15- \\
15 \text { reps at } 30 \% 1 \text { RM; BFR set to } 60 \% \text { arterial occlusion }\end{array}$ & $\begin{array}{l}\text { Pain reduction: } \mathrm{BFR}>\mathrm{HI} \text {. } \\
\text { Knee extensor torque: } \mathrm{BFR}>\mathrm{HI} \\
\text { for those with painful resisted knee } \\
\text { extension. } \\
\text { Worst pain, Kujala Patellofemoral } \\
\text { Score, pain with ADL, and knee } \\
\text { extensor torque: Improvements, } \\
\text { but no group differences }\end{array}$ \\
\hline $\begin{array}{l}\text { L. Hughes } \\
\text { et al., } 2018\end{array}$ & $\begin{array}{l}\mathrm{N}=30,76.7 \% \\
\text { male, } 29(7) \mathrm{yrs} \\
3 \text { weeks post-ACL } \\
\text { repair }\end{array}$ & $\begin{array}{l}\text { Partially randomized, } \\
\text { cross-sectional; } \\
\text { Groups: non-injured } \\
\text { resistance exercise, } \\
\text { ACL repair LI-BFR, } \\
\text { ACL repair HI }\end{array}$ & $\begin{array}{l}1 \text { session: Non-injured and BFR groups did } 4 \text { sets } 30- \\
15-15-15 \text { reps of unilateral leg press at } 30 \% 1 \mathrm{RM} \text {. ACL } \\
\text { repair heavy load exercise did } 3 \times 10 \text { reps at } 70 \% 1 \mathrm{RM} \text {. } \\
\text { BFR at } 80 \% \text { arterial occlusion }\end{array}$ & $\begin{array}{l}\text { RPE: BFR > non-injured. } \\
\text { Muscle pain: BFR }>A C L \text { repair } \\
\text { heavy load. } \\
\text { Knee pain: } B F R<A C L \text { repair } \\
\text { heavy load exercise }\end{array}$ \\
\hline
\end{tabular}




\begin{tabular}{|c|c|c|c|c|}
\hline 1 & 2 & 3 & 4 & 5 \\
\hline $\begin{array}{l}\text { D.J. Tennent } \\
\text { et al., } 2017\end{array}$ & $\begin{array}{l}\mathrm{N}=17,71 \% \text { men, } \\
37(7) \text { yrs, post } \\
\text { knee arthroscopy }\end{array}$ & $\begin{array}{l}\text { Pilot RCT; Groups: } \\
\text { BFR augmented PT, } \\
\text { regular PT }\end{array}$ & $\begin{array}{l}12 \text { training sessions; BFR: added additional } 3 \text { exercise } \\
\text { of leg press, extension, and reverse press to regular PT } \\
\text { regime for } 30-15-15-15 \text { reps at } 30 \% 1 \text { RM; Control group } \\
\text { did not perform the } 3 \text { additional exercise; BFR set } \\
\text { at } 80 \% \text { arterial occlusion }\end{array}$ & $\begin{array}{l}\text { Thigh girth: BFR > PT. } \\
\text { Stair climb: BFR > PT. } \\
\text { KOOS, other physical function } \\
\text { tests: both groups improved, } \\
\text { but no significant group difference } \\
\text { VR12: BFR > PT. } \\
\text { Strength: BFR > PT }\end{array}$ \\
\hline \multicolumn{5}{|c|}{ Athletic population } \\
\hline $\begin{array}{l}\text { C.J. Cook } \\
\text { et al., } 2014\end{array}$ & $\begin{array}{l}\mathrm{N}=20,100 \% \\
\text { male, } 21.5(1.4) \text { yrs, } \\
\text { semiprofessional } \\
\text { rugby players }\end{array}$ & $\begin{array}{l}\text { Cross-over design; } \\
\text { training: BFR, } \\
\text { standard training }\end{array}$ & $\begin{array}{l}\text { 3-wk training blocks; Both groups performed squat, } \\
\text { bench press, and weighted pull-up at } 70 \% 1 \mathrm{RM} ; 5 \times 5 \\
\text { reps with } 90 \text {-sec rest between sets, 3-min rest between } \\
\text { exercises; BFR set at } 180 \mathrm{mmHg}\end{array}$ & $\begin{array}{l}\text { Squat: BFR > standard. } \\
\text { Bench press: BFR > standard. } \\
\text { Sprint time improvement: BFR > } \\
\text { standard. } \\
\text { Testosterone and cortisol } \\
\text { responses: BFR > standard }\end{array}$ \\
\hline $\begin{array}{l}\text { T.W. Godawa } \\
\text { et al., } 2012\end{array}$ & $\begin{array}{l}\mathrm{N}=18,78 \% \\
\text { male, } 21.5(2.3) \\
\text { yrs, competitive } \\
\text { collegiate power } \\
\text { lifters }\end{array}$ & $\begin{array}{l}\mathrm{RCT} \text {; groups: BFR, } \\
\text { control }\end{array}$ & $\begin{array}{l}\text { 10-wk training following detailed staggered linear } \\
\text { progression model with wave pattern of progression for } \\
\text { all relevant lifts in both groups (squat, deadlifts, bench } \\
\text { press); Groups completed identical training with the only } \\
\text { difference being BFR; BFR pressure was personalized } \\
\text { and determined in supine position }\end{array}$ & $\begin{array}{l}\text { Squat training volume: BFR > } \\
\text { Control. } \\
\text { Squat strength: BFR > Control. } \\
\text { Deadlift, bench press, or total } \\
\text { score: Improvement, but no } \\
\text { significant group differences }\end{array}$ \\
\hline
\end{tabular}

\section{Uninjured young adults}

Studies involving uninjured young adults had in total 147 participants. The majority of these studies focused on outcomes for hypertrophy and strength, rather than function. The functional aspect was related to the training methods used in each study, which included exercises such as leg press, bench press, isometric handgrip - which has been associated with improving muscle function and blood flow (McGowan et al., 2007; Shephard, Montelpare, Plyley, McCracken, Goode, 1991) - and stepping. Five studies incorporating BFR training with the uninjured young adult population demonstrated that BFR training significantly increased the strength of the involved musculature (Credeur et al., 2010; Madarame et al., 2011; Ozaki et al., 2013; Teramoto, Golding, 2006; Yasuda et al., 2010). However, two of these studies showed no difference between BFR and the control groups, suggesting that low-load BFR training may result in similar results to traditional resistance training (Credeur et al., 2010; Ozaki et al., 2013). In the study by D.P. Credeur et al. (2010), participants performed identical exercise protocols using an isometric handgrip, with the inter group difference being the added BFR; thus, a group difference should have been likely. In contrast, participants in the H. Ozaki et al. (2013) study trained with either low intensity and BFR or at a high intensity without BFR. Group differences would be less likely in this scenario because the added benefit of BFR with low intensity work creates similar physiological effects to high intensity exercise. However, this is difficult to truly decipher without details to compare training volume differences between the groups. Three studies found that the addition of BFR was more effective in increasing strength than traditional resistance training of the same intensity and volume (Madarame et al., 2011; Teramoto, Golding, 2006; Yasuda et al., 2010). BFR was also found to significantly increase the cross-sectional area of the involved musculature (Credeur et al., 2010; Ozaki et al., 2013; Teramoto, Golding, 2006; Yasuda et al., 2010), yet studies were divided regarding the lack difference from traditional resistance training groups (Ozaki et al., 2013; Teramoto, Golding, 2006), and BFR training resulting in a larger increase in cross-sectional area of musculature than traditional resistance training (Credeur et al., 2010; 
Yasuda et al., 2010). Collectively, two studies from T. Yasuda et al. demonstrated that BFR training was less effective in increasing strength and cross-sectional area than high-intensity resistance training (Yasuda, Ogasawara, Sakamaki, Bemben, Abe, 2011; Yasuda et al., 2011b). Each study in this section utilized an absolute occlusion pressure, apart from M. Teramoto et al. (2006), which used a relative occlusion pressure based on the midpoint between systolic and diastolic blood pressures. The remaining studies in this group utilized pressures as low as $80 \mathrm{~mm} \mathrm{Hg}$ with no change throughout the study (Credeur et al., 2010), beginning at $100 \mathrm{~mm} \mathrm{Hg}$ and increasing to $160 \mathrm{~mm} \mathrm{Hg}$ with training (Ozaki et al., 2013; Yasuda et al., 2010; Yasuda et al., 2011a; Yasuda et al., 2011b), or beginning at $200 \mathrm{~mm} \mathrm{Hg}$ and increasing to $250 \mathrm{~mm} \mathrm{Hg}$ with training (Madarame et al., 2011).

\section{Oldep adults}

The total studies involving older adults (mean age below $65 \mathrm{yrs}$ ) consisted of a total of 117 participants. Two relevant studies incorporating BFR training with an older adult population demonstrated that low-load BFR training significantly increased the strength of the involved musculature (Araujo et al., 2015; Segal, N. et al., 2015). Both of these studies utilized the same low-load (30\% 1-RM) training protocol for two exercise groups with one using BFR. N.A. Segal et al.'s (2015) study of older women with osteoarthritis (OA) was the only study that showed significant improvement over low-load resistance training without BFR. Interestingly, N. Segal et al.'s (2015) similar study of older men with OA showed no significant improvement in strength following BFR training, and both groups performed identical training with the only exception being the added benefit of BFR for one group. Studies demonstrated improvements in functional outcome measures such as the timed up and go (TUG), sit to stand (STS), and stair limb (Araujo et al., 2015; Segal, N. et al., 2015). But only Araújo et al.'s (2015) study demonstrated that the BFR training group had significant functional improvement over the non-BFR group. Interestingly, Araújo et al. (2015) used a relative occlusion pressure at $80 \%$ of arterial occlusion, and the two studies by N. Segal et al. (Segal, N.A. et al., 2015) used an absolute pressure starting at $30 \mathrm{~mm} \mathrm{Hg}$ and increasing up to $200 \mathrm{~mm} \mathrm{Hg}$ with training.

\section{Rehabilitation}

Studies involving those undergoing rehabilitation involved a total of 116 participants. Three relevant studies incorporating BFR training with the rehabilitation population demonstrated that BFR training significantly increased strength and reduced pain from the injury after the session or training period (Giles et al., 2017; Hughes et al., 2018; Tennent et al., 2017). L. Giles et al. (2017) reported this significant benefit of low intensity BFR training over high intensity resistance training without BFR in patients with patellofemoral pain. Both studies with ACL reconstruction rehabilitation patients showed less knee pain with low-load BFR training compared to high-load traditional training. D.J. Tennant et al. (2017) also reported increased function and perceived health and improvements in strength compared to standard physical therapy care. Changes in cross-sectional area for this population was equivocal as D.J. Tennant et al. (2017) has demonstrated an improvement over traditional training, while L. Giles et al. (2017) demonstrates less improvement. However, the BFR group in Tennet et al. performed extra exercises compared to the control group. All three studies utilized relative occlusion set at $60 \%$ (Giles et al., 2017) or $80 \%$ arterial occlusion (Hughes et al., 2018; Tennent et al., 2017). 


\section{Athletes}

Studies involving athletes included a total of 38 participants. The two studies showed that incorporating BFR training with an athletic population significantly increased strength and power. Both studies were conducted with training groups performing identical training volume, with the only difference being the addition of BFR. T.M. Godawa et al. (2012) only demonstrated improved strength in squats with no group differences in deadlift or bench press, and C.J. Cook et al. (2014) was able to demonstrate that BFR training significantly improved strength and power over traditional training with the ability to maintain sprint performance longer. Further, Cook et al. (2014) showed an increased testosterone and cortisol response in the BFR group compared to the control, where BFR was set at an absolute arterial occlusion pressure of $180 \mathrm{~mm} \mathrm{Hg}$ throughout the three weeks of training. T.M. Godawa et al. (2012) utilized a personalized method and varied cuff pressures throughout training, but details regarding the pressure are not reported.

\section{Discussion}

Current literature suggests that functional BFR resistance training is effective, with more than $80 \%$ of the studies analyzed showing strength gains (Araujo et al., 2015; Cook et al., 2014; Credeur et al., 2010; Giles et al., 2017; Godawa et al., 2012; Madarame et al., 2011; Ozaki et al., 2013; Tennent et al., 2017; Teramoto, Golding, 2006; Yasuda et al., 2010). Of those studies, nearly two-thirds reported that BFR training was more effective than the control exercise group who trained at identical intensity and repetition ranges (Cook et al., 2014; Godawa et al., 2012; Madarame et al., 2011; Segal, N. et al., 2015; Tennent et al., 2017; Teramoto, Golding, 2006; Yasuda et al., 2010). The majority of the studies that compared low intensity BFR training to a more traditional high intensity training regime showed no differences between the groups in regard to strength and hypertrophy. Nine of the studies included in this review measured changes in muscle size, with all of them showing a significant increase in size with functional BFR training (Credeur et al., 2010; Madarame et al., 2011; Ozaki et al., 2013; Segal, N. et al., 2015; Tennent et al., 2017; Teramoto, Golding, 2006; Yasuda et al., 2011a; Yasuda et al., 2011b). Two of these studies showed a greater change in muscle size in high-intensity training compared to the low intensity BFR training (Yasuda et al., 2010; Yasuda et al., 2011b).

It seems that many studies used different BFR protocols in the training sessions. Most studies set the intensity for functional BFR at 20-30\% 1RM, in accord with previous studies to date (Hughes et al., 2018; Madarame et al., 2011; Ozaki et al., 2013; Segal, N. et al., 2015; Segal, N.A. et al., 2015; Yasuda et al., 2010; Yasuda et al., 2011a; Yasuda et al., 2011b). Many also chose to use a training pattern of one set of 30 repetitions followed by three sets of 15 repetitions with 30 seconds rest (Araujo et al., 2015; Giles et al., 2017; Hughes et al., 2018; Ozaki et al., 2013; Segal, N. et al., 2015; Segal, N.A. et al., 2015; Tennent et al., 2017; Yasuda et al., 2010; Yasuda et al., 2011a; Yasuda et al., 2011b). Some studies set an absolute occlusion pressure (Cook et al., 2014; Credeur et al., 2010), others used a prescribed incremental protocol to raise the pressure during an "acclimation period" (Madarame et al., 2011; Ozaki et al., 2013; Segal, N. et al., 2015; Segal, N.A. et al., 2015; Yasuda et al., 2010; Yasuda et al., 2011a; Yasuda et al., 2011b), but many chose a relative occlusion pressure ranging from 60-80\% occlusion (Araujo et al., 2015; Giles et al., 2017; Hughes et al., 2018; Tennent et al., 2017). Studies that set their pressures lower than $100 \mathrm{mmHg}$ tended to have no difference between the experimental groups, probably not providing sufficient occlusion to stimulate the factors needed for muscular adaptation (Credeur et al., 2010; Teramoto, Golding, 2006). While there appears to 
be no consensus on occlusion prescription, setting the occlusion pressure relative to each person may be more appropriate to elicit improvements in strength and enhance safety for the exercising individual.

In the case of a clinical population who is unable to adhere to ACSM guidelines for traditional strength training, functional BFR may be an effective alternative to achieve similar if not better strength adaptations than traditional resistance training alone (Araujo et al., 2015; Cook et al., 2014; Credeur et al., 2010; Giles et al., 2017; Godawa et al., 2012; Madarame et al., 2011; Ozaki et al., 2013; Segal, N. et al., 2015; Tennent et al., 2017; Teramoto, Golding, 2006; Yasuda et al., 2010). Functional training with BFR was shown to be highly effective in a rehabilitation population who either experienced significant pain or were unable to perform high intensity functional training (Giles et al., 2017; Hughes et al., 2018; Tennent et al., 2017). This may be a key factor for decreasing the rehabilitation time for postinjury or post-surgery patients. The added benefit of BFR with functional exercises was also shown in the studies conducted with uninjured older adults (Araujo et al., 2015; Segal, N. et al., 2015).

In an athletic population, the study by Cook et al. (Cook et al., 2014) is unique as it was the first multi-joint BFR study to find significant strength gains in a short three-week training period. This time frame is exceptionally short for traditional strength gains which require about six weeks (Garber et al., 2011). This study incorporated BFR training with high intensity work, which may have led to quick gains, but more research is necessary to discern the physiological rationale for the improvements. The use of functional BFR training in athletes could provide some salient exercises required in the sport while reducing load to decrease risk of overtraining and injuries, while dynamic exercises may provide the best opportunity for neural adaptation (Madarame et al., 2011; Pelletier et al., 2015; Yasuda et al., 2011a; Yasuda et al., 2011b).

Current literature also suggests that functional BFR resistance training is overall effective in increasing muscle CSA in the involved musculature (Credeur et al., 2010; Ozaki et al., 2013; Tennent et al., 2017; Yasuda et al., 2010; Yasuda et al., 2011a; Yasuda et al., 2011b). Of these studies, 43\% report that functional BFR training was more effective than the control exercise group for increasing muscle CSA (Credeur et al., 2010; Tennent et al., 2017; Yasuda et al., 2011a). After comparing all relevant studies, it is difficult to make a strong conclusion, but most suggest that muscular strength gains are likely due to hypertrophy rather than neural adaptation and fiber recruitment patterns during BFR (Loenneke et al., 2012; Madarame et al., 2011; Segal, N. et al., 2015; Yasuda et al., 2011a; Yasuda et al., 2011b). It is important to understand the degree of neural adaptation in functional BFR training, as it contributes to the overall success and performance of the participants. Yasuda et al. (2010) suggests that BFR training only increases strength through hypertrophy without neural adaptation. Subsequently, combining functional BFR with high-intensity exercise might be best to achieve maximal strength gains through both hypertrophy and neural adaptation (Yasuda et al., 2011a). This is similar to the conclusion from a previous BFR systematic review that reported superior muscular adaptations in high-load resistance training due to the contribution of neural adaptations and proper muscle fiber firing (Lixandrao et al., 2018). The present study did not assess neural changes in the exercised muscle groups.

When applying functional BFR to a clinical population, improvements in neural adaptations to ensure proper function should not be neglected. This is especially important in treating chronic conditions because the restoration of proper motor activity through neuroplasticity principles are necessary to encourage proper functioning that continues after rehabilitation (Pelletier et al., 2015). Functional BFR training has positive effects on functional outcomes, functional performance, pain reduction and quality of life (Araujo et al., 2015; Cook et al., 2014; Giles 
et al., 2017; Godawa et al., 2012; Hughes et al., 2018; Tennent et al., 2017). Tennent et al. (2017) was the only study that performed a subjective outcome measure to analyze the perspective of wellness and quality of life, which also improved significantly with the use of functional BFR. Future research utilizing more subjective measures of the success of functional BFR in the perspective of the participant would help to determine its effect on life participation rather than performance. Literature is lacking in studies that provide self-reported quality of life and pain after functional BFR training, which is limiting its clinical utility. Of the three studies that analyzed pain after the session in the rehabilitation group, all reported reduced pain after using functional BFR exercises (Giles et al., 2017; Hughes et al., 2018; Tennent et al., 2017). Hughes et al. (2018) suggest that using light loads during early rehabilitation will prevent further inflammation, and will lead to improved outcomes in self-reported function and overall pain.

\section{Conclusion}

Current relevant literature suggests that functional BFR exercise training is successful in improving strength, hypertrophy, pain reduction, and function in injured and uninjured populations of various ages. Clinicians who wish to use functional BFR training should be aware that strength improvements may be more from increased muscle hypertrophy than from neural adaptations. Choosing occlusion pressures for effectiveness is difficult as the studies showed great variation. Utilizing a relative pressure of $60 \%$ arterial occlusion or greater would likely create the advantageous hypoxic environment necessary to induce the desired physiological response. More research is necessary to understand the effect of BFR functional training on these neural adaptations, self-reported outcomes, hypertrophy in athletic and older adults, and the musculature adaptations that occur in those that are unconcluded.

\section{Referenences}

Araujo, J.P., Neto, G.R., Loenneke, J.P., Bemben, M.G., Laurentino, G.C., Batista, G., Silva, J.C.G., Freitas, E.D.S, Sousa, M.S.C. (2015). The effects of water-based exercise in combination with blood flow restriction on strength and functional capacity in postmenopausal women. Age (Dordr), 37 (6), 110.

Brandner, C.R., May, A.K., Clarkson, M.J., Warmington, S.A. (2018). Reported side-effects and safety considerations for the use of blood flow restriction during exercise in practice and research. Techniques in Orthopaedics, 33 (2), 1.

Clarkson, M.J., Fraser, S.F., Bennett, P.N., McMahon, L.P., Brumby, C., Warmington, S.A. (2017). Efficacy of blood flow restriction exercise during dialysis for end stage kidney disease patients: Protocol of a randomised controlled trial. BMC Nephrology, 18 (1). DOI: 10.1186/s12882-017-0713-4.

Cook, C.J., Kilduff, L.P., Beaven, C.M. (2014). Improving strength and power in trained athletes with 3 weeks of occlusion training. International Journal of Sports Physiology and Performance, 9 (1), 166-172. DOI: 10.1123/ijspp.2013-0018.

Credeur, D.P., Hollis, B.C., Welsch, M.A. (2010). Effects of handgrip training with venous restriction on brachial artery vasodilation. Medicine and Science in Sports and Exercise, 42 (7), 1296-1302. DOI: 10.1249/MSS.0b013e3181ca7b06.

Garber, C.E., Blissmer, B., Deschenes, M.R., Franklin, B.A., Lamonte, M.J., Lee, I.M., Nieman, D.C., Swain, D.P. (2011). American college of sports medicine position stand. Quantity and quality of exercise for developing and maintaining cardiorespiratory, musculoskeletal, and neuromotor fitness in apparently healthy adults: Guidance for prescribing exercise. Medicine and Science in Sports and Exercise, 43 (7), 1334-1359. DOI: 10.1249/MSS.0b013e318213fefb.

Giles, L., Webster, K.E., McClelland, J., Cook, J.L. (2017). Quadriceps strengthening with and without blood flow restriction in the treatment of patellofemoral pain: A double-blind randomised trial. British Journal of Sports Medicine, 51 (23), 1688-1694. DOI: 10.1136/bjsports-2016-096329.

Godawa, T.M., Credeur, D.P., Welsch, M.A. (2012). Influence of compressive gear on powerlifting performance: Role of blood flow restriction training. Journal of Strength and Conditioning Research, 26 (5), 1274-1280. DOI: 10.1519/JSC.0b013e3182510643. 
Hughes, L., Paton, B., Haddad, F., Rosenblatt, B., Gissane, C., Patterson, S.D. (2018). Comparison of the acute perceptual and blood pressure response to heavy load and light load blood flow restriction resistance exercise in anterior cruciate ligament reconstruction patients and non-injured populations. Physical Therapy in Sport: Official Journal of the Association of Chartered Physiotherapists in Sports Medicine, 33, 54-61. DOI: S1466-853X(18)30175-5.

Lixandrao, M.E., Ugrinowitsch, C., Berton, R., Vechin, F.C., Conceicao, M.S., Damas, F., Libardi, C.A., Roschel, H. (2018). Magnitude of muscle strength and mass adaptations between high-load resistance training versus low-load resistance training associated with blood-flow restriction: A systematic review and meta-analysis. Sports Medicine (Auckland, N.Z.), 48 (2), 361-378. DOI: 10.1007/s40279-017-0795-y.

Loenneke, J.P., Wilson, J.M., Marin, P.J., Zourdos, M.C., Bemben, M.G. (2012). Low intensity blood flow restriction training: A metaanalysis. European Journal of Applied Physiology, 112 (5), 1849-1859. DOI: 10.1007/s00421-011-2167-x.

Madarame, H., Ochi, E., Tomioka, Y., Nakazato, K., Ishii, N. (2011). Blood flow-restricted training does not improve jump performance in untrained young men. Acta Physiologica Hungarica, 98 (4), 465-471. DOI: 10.1556/APhysiol.98.2011.4.10.

McCully, K.K., Smith, S., Rajaei, S., Leigh, J.S. Jr, Natelson, B.H. (2003). Blood flow and muscle metabolism in chronic fatigue syndrome. Clinical Science, 104 (6). 641-647. DOI: 10.1042/CS20020279.

McGowan, C.L., Visocchi, A., Faulkner, M., Verduyn, R., Rakobowchuk, M., Levy, A.S. McCartney, N., MacDonald, M.J. (2007). Isometric handgrip training improves local flow-mediated dilation in medicated hypertensives. European Journal of Applied Physiology, 99 (3), 227-234. DOI: 10.1007/s00421-006-0337-z.

Ozaki, H., Yasuda, T., Ogasawara, R., Sakamaki-Sunaga, M., Naito, H., Abe, T. (2013). Effects of high-intensity and blood flow-restricted low-intensity resistance training on carotid arterial compliance: Role of blood pressure during training sessions. European Journal of Applied Physiology, 113 (1), 167-174. DOI: 10.1007/s00421-012-2422-9.

Patterson, S.D., Hughes, L., Warmington, S., Burr, J., Scott, B.R., Owens, J., Abe, T., Nielsen, J.L., Libardi, C., Laurentino, G., Neto, G., Brandner, C., Martin-Hernandez, J., Loenneke, J. (2019). Blood flow restriction exercise position stand: Considerations of methodology, application, and safety. Frontiers in Physiology, 10, 533.

Pelletier, R., Higgins, J., Bourbonnais, D. (2015). Is neuroplasticity in the central nervous system the missing link to our understanding of chronic musculoskeletal disorders? BMC Musculoskeletal Disorders, 16. DOI: 10.1186/s12891-015-0480-y.

Segal, N., Davis, M.D., Mikesky, A.E. (2015). Efficacy of blood flow-restricted low-load resistance training for quadriceps strengthening in men at risk of symptomatic knee osteoarthritis. Geriatric Orthopaedic Surgery \& Rehabilitation, 6 (3), 160-167. DOI: $10.1177 / 2151458515583088$.

Segal, N.A., Williams, G.N., Davis, M.C., Wallace, R.B., Mikesky, A.E. (2015). Efficacy of blood flow-restricted, low-load resistance training in women with risk factors for symptomatic knee osteoarthritis. PM \& R: The Journal of Injury, Function, and Rehabilitation, 7 (4), 376-384. DOI: 10.1016/j.pmrj.2014.09.014.

Shephard, R.J., Montelpare, W., Plyley, M., McCracken, D., Goode, R.C. (1991). Handgrip dynamometry, cybex measurements and lean mass as markers of the ageing of muscle function. British Journal of Sports Medicine, 25 (4), 204-208. DOI: 10.1136/ bjsm.25.4.204.

Takarada, Y., Takazawa, H., Sato, Y., Takebayashi, S., Tanaka, Y., Ishii, N. (2000). Effects of resistance exercise combined with moderate vascular occlusion on muscular function in humans. Journal of Applied Physiology (Bethesda, Md.: 1985), 88 (6), 2097-2106. DOI: 10.1152/jappl.2000.88.6.2097.

Takarada, Y., Tsuruta, T., Ishii, N. (2004). Cooperative effects of exercise and occlusive stimuli on muscular function in low-intensity resistance exercise with moderate vascular occlusion. The Japanese Journal of Physiology, 54 (6), 585-592. DOI: 10.2170/ jjphysiol.54.585.

Tennent, D.J., Hylden, C.M., Johnson, A.E., Burns, T.C., Wilken, J.M., Owens, J.G. (2017). Blood flow restriction training after knee arthroscopy: A randomized controlled pilot study. Clinical Journal of Sport Medicine: Official Journal of the Canadian Academy of Sport Medicine, 27 (3), 245-252. DOI: 10.1097/JSM.0000000000000377.

Teramoto, M., Golding, L.A. (2006). Low-intensity exercise, vascular occlusion, and muscular adaptations. Research in Sports Medicine (Print), 14 (4), 259-271. DOI: 10.1080/15438620600985860.

Yasuda, T., Fujita, S., Ogasawara, R., Sato, Y., Abe, T. (2010). Effects of low-intensity bench press training with restricted arm muscle blood flow on chest muscle hypertrophy: A pilot study. Clinical Physiology and Functional Imaging, 30 (5), 338-343. DOI: 10.1111/j.1475-097X.2010.00949.X. 
Yasuda, T., Ogasawara, R., Sakamaki, M., Bemben, M.G., Abe, T. (2011a). Relationship between limb and trunk muscle hypertrophy following high-intensity resistance training and blood flow-restricted low-intensity resistance training. Clinical Physiology and Functional Imaging, 31 (5), 347-351. DOI: 10.1111/j.1475-097X.2011.01022.x.

Yasuda, T., Ogasawara, R., Sakamaki, M., Ozaki, H., Sato, Y., Abe, T. (2011b). Combined effects of low-intensity blood flow restriction training and high-intensity resistance training on muscle strength and size. European Journal of Applied Physiology, 111 (10), 2525-2533. DOI: 10.1007/s00421-011-1873-8.

Cite this anticle aS: Hernandez, N.J., Myers, B.J., Feito, Y., Bunn, J.A. (2020). Functional Strength Exercise and Blood-flow Restriction Protocols: A Systematic Review. Central European Journal of Sport Sciences and Medicine, 3 (31), 47-58. DOI: 10.18276/cej.2020.3-04. 